\title{
Neurogénesis hipocampal adulta y envejecimiento cognitivo
}

\section{Adult hippocampal neurogenesis and cognitive aging}

\author{
Román Darío Moreno Fernández ${ }^{1,2}$, Carmen Pedraza ${ }^{2}$ y Milagros Gallo ${ }^{1}$ \\ ${ }^{1}$ Departmento de Psicobiología. Instituto de Neurociencias. Centro de Investigaciones Biomédicas (CIBM). \\ Universidad de Granada, España. \\ ${ }^{2}$ Departamento de Psicobiología y Metodología en CC. Universidad de Málaga. Instituto de Investigaciones Biomédicas \\ de Málaga (IBIMA), España.
}

Disponible online 31 de diciembre de 2013

\begin{abstract}
El envejecimiento es un proceso normal en el desarrollo del organismo asociado a una serie de cambios neurobiológicos que producen alteraciones cognitivas con funciones preservadas, deterioradas y facilitadas. Se revisa la evidencia obtenida con animales y humanos a fin de explorar un posible papel de la plasticidad hipocampal en los cambios cognitivos asociados con la edad, con especial atención a la neurogénesis hipocampal adulta. Los resultados obtenidos empleando las estrategias de lesión, estimulación, así como datos correlacionales apoyan un papel, ya sea directo, ya sea modulador de las nuevas neuronas en la ejecución cognitiva a edades avanzadas. Avances en la investigación de esta relación pueden favorecer el desarrollo de nuevos tratamientos y la mejora de la calidad de vida de la población de más edad.
\end{abstract}

Palabras clave: Neurogénesis hipocampal adulta; Envejecimiento; Deterioro Cognitivo; Memoria Espacial.

Aging is a normal developmental process associated with neurobiological changes leading to cognitive alterations with preserved, impaired, and enhanced functions. Evidence from animal and human studies is reviewed to explore the potential role of hippocampal plasticity on age-related cognitive changes with special attention to adult hippocampal neurogenesis. Results from lesion and stimulation strategies, as well as correlation data, support either a direct or modulatory role for adult newborn neurons in cognition at advanced ages. Further research on this topic may help to develop new treatments and to improve the quality of life of older people.

Key words: Adult Hippocampal Neurogenesis; Aging; Cognitive Decline; Spatial Memory.

Correspondencia: Román Darío Moreno Ferrnández. Instituto de Investigaciones Biomédicas de Málaga (IBIMA). Departamento de Psicobiología y Metodología en CC Facultad de Psicología. Campus de Teatinos, 29071 Málaga. E-mail: roman_amyc@hotmail.com. E-mail de los otros autores: Carmen Pedraza: mdpedraza@uma.es, Milagros Gallo: mgallo@ugr.es

Los autores agradecen la contribución del proyecto de investigación PSI2011-23702 (MINECO, Spain, financiado parcialmente con fondos FEDER). 
El envejecimiento es un proceso normal en la vida del organismo en el que se combinan la necesidad de adaptación a nuevos retos impuestos por modificaciones de las capacidades biológicas, junto a los efectos de varias décadas de historia personal. Frecuentemente el deterioro ocasionado por procesos patológicos a lo largo de la vida y la mayor susceptibilidad a diversas patologías propias de la edad avanzada hacen difícil disociar los cambios asociados al envejecimiento denominado "normal" "saludable" o "satisfactorio" de aquellos relacionados con procesos patológicos que dan lugar al denominado “envejecimiento patológico". Ello es especialmente cierto en el estudio de las alteraciones cognitivas inducidas por la edad que resultan difícil de distinguir, en ocasiones, de los síntomas tempranos de trastornos neurodegenerativos como la enfermedad de Alzheimer. No obstante, tanto análisis neuropsicológicos de la ejecución en personas de edad avanzada que permiten establecer diferencias sutiles y facilitan el diagnóstico diferencial como el empleo de modelos animales que carecen de patologías (Gallagher y Rapp, 1997) resultan de especial importancia para el desarrollo de nuevos tratamientos y avances en el conocimiento para la mejora de la calidad de vida de la población de más edad.

La dificultad en disociar envejecimiento normal y patológico ha inclinado a una visión del envejecimiento con especial hincapié en el deterioro o decaimiento de las funciones que hoy está siendo revisada (Reuter-Lorenz y Lustig, 2005). En efecto, incluso en ausencia de patologías, dadas las propiedades plásticas del sistema nervioso, lo esperable es que se produzcan modificaciones adaptativas a lo largo de la vida en su organización funcional dando lugar a cambios en el comportamiento $\mathrm{y}$, específicamente, en las funciones cognitivas. Las características de los cambios cognitivos asociados al envejecimiento apoyan dicho planteamiento. En primer lugar, se ha observado que dichas modificaciones incluyen tanto declive de determinadas funciones cognitivas, mientras que otras resultan preservadas e incluso facilitadas con la edad (Gámiz y Gallo, 2011). Aunque la mejora o potenciación de determinadas funciones ha sido interpretada frecuentemente en términos de compensación de funciones deterioradas, es evidente la existencia de reorganización incompatible con un mero deterioro global. En segundo lugar, los cambios son paulatinos iniciándose mucho antes de la senectud. En algunas de las funciones que pueden sufrir deterioro, éste puede iniciarse a edades relativamente tempranas y progresar lentamente mientras que en otras no se hace evidente hasta etapas más tardías (Junqué y Jurado 1994). Ello se interpreta en términos de una susceptibilidad diferencial al deterioro inducido por la edad, pero no puede descartarse que la pérdida de determinadas funciones que han dejado de ser necesarias resulte una modificación adaptativa. En tercer lugar, la variabilidad en las alteraciones cognitivas existente a edades avanzadas es una constante tanto en la población humana que no sufre patologías como en modelos animales (Foster, 2012). Ello conduce a disociar edad cronológica versus edad "cogni- tiva", lo que puede relacionarse con el impacto acumulado de las experiencias individuales a lo largo de la vida. El deterioro de aquellas funciones más susceptibles a los efectos de la edad no es inevitable, ya que no aparece en todos los individuos. En este sentido, existen numerosos datos que ponen en evidencia el efecto de experiencias tempranas desde etapas fetales y neonatales del desarrollo sobre la ejecución cognitiva a edades avanzadas que alteran a su vez la plasticidad neural (Lemaire, Koehl, Le Moal y Abrous, 2000, 2006; Meaney, Aitken, Bhatnagar y Sapolsky, 1991).

Con el fin de explorar la relación entre los cambios cognitivos asociados a la edad y los procesos de plasticidad neural subyacentes esta revisión se centra en las funciones que dependen del sistema temporal con especial énfasis en el hipocampo, uno de los sistemas implicados en aprendizaje y memoria más susceptibles al envejecimiento (Lister y Barnes, 2009). Además, el hipocampo ha resultado de especial interés como sistema modelo en la investigación tanto de los mecanismos de plasticidad sináptica responsables de fenómenos tales como la potenciación y depresión a largo plazo, como de la generación de nuevas neuronas durante la vida adulta. Dada la gran cantidad de datos existentes sobre la función hipocampal, ejecución cognitiva y envejecimiento, esta revisión no pretende ser exhaustiva, sino explorar una posible participación del proceso de neurogénesis hipocampal adulta en el proceso de envejecimiento cognitivo; entendiendo este último como el conjunto de modificaciones en las funciones cognitivas asociadas a la edad avanzada. Para ello se revisarán tanto los datos obtenidos en población humana como en modelos animales.

\section{Envejecimiento cognitivo}

En este sentido, se ha descrito una variedad de procesos cognitivos susceptibles al efecto de la edad, incluyendo las funciones ejecutivas y la memoria de trabajo, asociadas típicamente a una función alterada de la corteza prefrontal (Wang et al., 2011), la claridad y eficiencia del procesamiento (Goh y Park, 2009; Head, Rodriguez, Kennedy y Raz, 2008; Turner y Spreng, 2012) y la cognición social, especialmente en entornos complejos (Kemp, Després, Sellal y Dufour, 2012). Ello se ha asociado con cambios en la organización anatómico-funcional de la zona (Burke y Barnes, 2006; Lister y Barnes, 2009) tales como disminución del número y longitud de dendritas, menor número de axones y deterioro mielínico así como pérdida sináptica importante (Pannese, 2011; Salat, 2011). Asimismo, se ha descrito una reducción significativa del volumen e integridad de la sustancia blanca en personas mayores (Caserta et al., 2009). Una revisión sistemática de los resultados empleando técnicas de neuroimagen (Spreng, Wojtowicz y Grady, 2010) indica mayor actividad prefrontal en adultos mayores frente a los jóvenes en diversos dominios cognitivos, incluyendo percepción, función ejecutiva y memoria, incluso siendo la ejecución similar. La existencia de patrones de activación hiperfrontales asociados al envejecimiento ha sido interpretada frecuentemente 
en términos de compensación para paliar el malfuncionamiento de circuitos funcionales en el adulto, especialmente el sistema temporal (Jagust, 2013). Efectivamente las deficiencias cognitivas más frecuentemente asociadas al envejecimiento afectan al aprendizaje y la memoria dependientes del hipocampo y áreas temporales asociadas, siendo más afectada la memoria semántica y episódica de hechos recientes y de poca relevancia para el individuo, mientras que resulta preservada la memoria remota así como la memoria implícita (Rieckmann y Bäckman, 2009) y la memoria emocional (Hedden y Gabrielli, 2004). Del mismo modo se ha descrito deterioro en tareas dependientes del hipocampo que implican memoria espacial y complejidad empleando procedimientos de miedo condicionado y condicionamiento palpebral (Cansino, 2009; Craik y Rose, 2012; Jessberger y Gage, 2008; Lithfous, Dufour y Després, 2013).

Resultados similares se han obtenido en modelos animales (para una revisión reciente véase Samson y Barnes, 2013) siendo las tareas más utilizadas la piscina de Morris en roedores, desemparejamiento demorado con la muestra en primates no humanos, memoria de reconocimiento de objetos complejos (Burke, Wallace, Uprety y Barnes, 2010; Gámiz y Gallo, 2012), condicionamiento del miedo al contexto y condicionamiento de huella palpebral en roedores (Alexander et al., 2012). Por otra parte, el empleo de tareas de aprendizaje y memoria gustativa en roedores muestran una compleja reorganización durante el envejecimiento con funciones que no decaen con la edad, mientras que otras resultan potenciadas (Morón y Gallo, 2007) o deterioradas (revisado en Gámiz y Gallo, 201; Manrique, Morón, Ballesteros, Guerrero y Gallo, 2007; Morón, Ballesteros, Cándido y Gallo, 2002). Los resultados confirman que resultan selectivamente afectadas aquellas funciones complejas dependientes del hipocampo, tales como el fenómeno de bloqueo condicionado, haciéndose evidente el deterioro en animales maduros antes de poder ser considerados envejecidos (Gallo, Valouskova y Cándido, 1997; Morón, Ballesteros, Valouskova y Gallo, 2001). Sin embargo, el efecto de la lesión hipocampal en ratas envejecidas no induce efectos similares a dicha lesión en animales jóvenes cuando se utiliza tareas de memoria gustativa dependiente del contexto temporal. Ello indica que la función moduladora hipocampal sobre la memoria gustativa sufre modificaciones a lo largo de la vida, por lo que un hipocampo envejecido no puede identificarse en modo alguno con un hipocampo dañado (Manrique et al., 2009).

Por lo tanto, las alteraciones en las funciones cognitivas no son generalizadas sino que afectan selectivamente a dominios específicos mostrando también una gran variabilidad entre los individuos. Para explicar la causa de dicha variabilidad se han propuesto diversas hipótesis. Tal es el ejemplo de la hipótesis de la retrogénesis de la sustancia blanca, que postula que las fibras de sustancia blanca mielinizadas tardíamente son las más vulnerables a la degeneración relacionada con la edad y la enfermedad, que finalmente provocará el deterioro cognitivo (Brickman et al., 2012). Asimismo, el grupo de Park propuso en su Teoría del Andamiaje del Envejecimiento Cognitivo (STAC) que los cambios funcionales debidos a la edad forman parte del proceso vital de andamiaje cognitivo compensatorio como intento de aliviar los déficits cognitivos asociados al envejecimiento; entendiendo como andamiaje el proceso que implica el uso y desarrollo de circuitos neurales complementarios y alternativos para alcanzar una meta cognitiva (Goh y Park, 2009; Park y Bischof, 2013; Park y Reuter-Lorenz, 2009). Además, otro de los supuestos planteados en este ámbito es la hipótesis de la reserva cognitiva que defiende que las diferencias individuales en adaptabilidad y flexibilidad de las redes neurales subyacentes a la función cognitiva pueden permitir a unas personas lidiar mejor con los cambios cerebrales (Steffener y Stern, 2012).

\section{Sistema hipocampal y envejecimiento}

Las neuronas hipocampales se distribuyen en tres subregiones principales: células granulares en el giro dentado, y neuronas piramidales en CA1 y CA3. La organización funcional está basada en un circuito trisináptico en el cual: primeramente las dendritas de las células granulares del giro dentado reciben aferencias de la corteza entorrinal por medio de la vía perforante; en segundo lugar, las células granulares (fibras musgosas) envían eferencias a las neuronas piramidales de CA3; y en tercer lugar, las neuronas piramidales de CA3 y CA1 proyectan de vuelta hacia la corteza entorrinal mediante el subículo (Kempermann, 2012).

La formación hipocampal es una de las más estudiadas en envejecimiento en referencia al deterioro de funciones cognitivas, debido a que: a) existe bastante evidencia que documenta una serie de alteraciones y deterioros en la función, morfología y metabolismo del hipocampo asociado a la edad avanzada (Erickson, Miller y Roecklein, 2012; Jessberg y Gage, 2008; Lister y Barnes, 2009); b) daños y alteraciones en el funcionamiento del sistema hipocampal han sido típicamente asociados a deterioros cognitivos como problemas de memoria (Milner, 2005); c) en general, todas las pruebas que detectan daño hipocampal son sensibles a los efectos de la edad; y d) el hipocampo es muy susceptible a los efectos de la enfermedad de Alzheimer, isquemia/hipoxia y generación de ataques epilépticos, los tres trastornos neurológicos más comunes en edad avanzada (Patrylo y Williamson, 2007). Sin embargo, a pesar de que en un principio diversos estudios apuntaban a la existencia de pérdida significativa de neuronas hipocampales con el envejecimiento (Ball, 1977; Brody, 1955), el desarrollo de nuevos principios estereológicos permitió observar que la pérdida celular no es característica del envejecimiento normal (Burke y Barnes, 2006). A su vez, tampoco se han encontrado cambios significativos respecto a ramificación de dendritas en CA1, CA3 y subículo, e incluso en el giro dentado parece haber una mayor ramificación en ancianos en comparación con adultos (Burke y Barnes, 2006). Tampoco se han constatado diferencias significativas respecto a las propiedades electrofi- 
siológicas basales de las neuronas hipocampales como el potencial de membrana de reposo (Barnes, 1994). Por lo tanto, es de destacar que, más que un hipocampo estructuralmente lesionado, lo que supone el envejecimiento normal es un hipocampo cuya función y conectividad han sido alteradas.

Así pues, se ha observado una reducción de conexiones sinápticas, liberación de neurotransmisores y número de receptores; siendo afectados también los mecanismos intracelulares de plasticidad hipocampal, como muestran un deterioro de la potenciación a largo plazo (LTP) y un aumento de la depresión a largo plazo (LTD; Rosenzweig y Barnes, 2003; Lister y Barnes, 2009; Mendelsohn y Larrick, 2012). Por otro lado, se observa un aumento de la respuesta en las sinapsis conservadas, así como reorganización de las conexiones neuronales, que ha sido interpretado como mecanismos compensatorio para el mantenimiento de la función a pesar de la pérdida sináptica (Goh y Park, 2009). Métodos estereológicos han mostrado reducciones del $24 \%$ de las sinapsis axoespinales en las capas moleculares media e interna del giro dentado (Geinisman, de Toledo-Morrell, Morrell, Persina y Rossi, 1992), mientras que no se ha hallado una pérdida sináptica significativa en CA1 (Geinisman, Ganeshina, Yoshida, Berry, Disterhoft y Gallagher, 2004).

Una de las zonas hipocampales más afectada funcionalmente es CA3, de forma que el exceso de actividad de las neuronas piramidales en esta región impide una codificación normal de nueva información y ha sido vinculado con las características de la memoria en ratas envejecidas (Wilson et al., 2003, 2005). En este sentido, recientemente se han propuesto varias estrategias farmacológicas para regular este exceso de actividad y favorecer la función cognitiva de ratas envejecidas, mejorando su ejecución en tareas de memoria espacial (Koh, Haberman, Foti, McCown y Gallagher, 2010; Koh, Rosenzweig-Lipson y Gallagher, 2013). Además, debido a la conectividad de esta región con el giro dentado, primer receptor de aferencias corticales del circuito hipocampal, también queda afectada esta función de filtro y la estimulación de las células granulares de esta capa no producen cambios o suprimen los potenciales de campo en CA3 (Patrylo y Williamson, 2007). Por último, además de las alteraciones en la estructura, función y expresión genética de las células granulares de esta formación (Chawla y Barnes, 2007) otra de las alteraciones hipocampales inducidas por la edad hace referencia a las nuevas neuronas generadas en el giro dentado, tema abordado en la siguiente sección.

\section{Neurogénesis hipocampal adulta}

Recientemente en la historia de la neurociencia se ha asumido la existencia de división de nuevas neuronas en el sistema nervioso adulto (Gross, 2000), la cual fue demostrada primero en ratas (Altman y Das, 1965), luego en el cerebro de ave por el grupo de investigación de Nottebohm (Goldman y Nottebohm, 1983) y finalmente en humanos (Eriksson et al., 1998). Actualmente existe evidencia para múltiples especies (Kempermann,
2012) y, en mamíferos, los nichos neurogénicos parecen quedar limitados a la zona subranular del giro dentado del hipocampo y la zona subventricular de los ventrículos laterales, desde donde migran hacia el bulbo olfatorio (Ehninger y Kempermann, 2007).

En el proceso de neurogénesis han sido identificadas varias etapas, tales como proliferación, migración y diferenciación (Gage, 2000). Aunque existen diversas posturas acerca de la naturaleza de las células progenitoras en la zona subgranular hipocampal que pueden ser células radiales tipo glial denominadas también células tipo 1, células tipo B o troncales neuroepiteliales, se han identificado diferentes marcadores específicos del tipo de célula a lo largo del proceso que convierte a las células progenitoras adultas en neuronas hipocampales. Así las células progenitoras que inicialmente expresan GFAP (glial fibrillary acidic protein), Sox2 y Nestina, pasan a expresar PSA-NCAM (polysialic acid-neural cell adhesion molecule), DCX (doublecortin) y NeuroD cuando se convierten en células progenitoras temporalmente amplificadas, mientras que como neuronas inmaduras (Masiulis, Sanghee y Eisch, 2011) expresan NeuroD y Calretinina, para finalmente expresar NeuN y Calbindina cuando se convierten en neuronas más maduras. Estas células migran y se diferencian en las formas finales de las células granulares, que se integran en la circuitería hipocampal en la capa granular (Eisch, Cameron, Encinas, Meltzer y Ming, 2008). La integración de nuevas neuronas durante la adultez en el circuito hipocampal posee diversas funciones, desde sustitución de neuronas perdidas a modificaciones de la red hipocampal y su funcionalidad (Song, Christian, Ming y Song, 2012). Además, las neuronas inmaduras, antes de diferenciarse, también contribuyen a la plasticidad sináptica del giro dentado (Kempermann, 2012).

Así, varias funciones han sido asociadas a la formación de nuevas neuronas, aunque aún queda por dilucidar su verdadera contribución funcional (Snyder y Cameron, 2012). Dada su ubicación en el hipocampo, se ha relacionado típicamente con procesos de aprendizaje y memoria, en especial la memoria espacial y episódica. Así, se ha mostrado mediante experimentos la implicación de las nuevas neuronas en la adquisición flexible de relaciones espacio-temporales entre señales del entorno (Koehl y Abrous, 2011). De esta manera, se ha propuesto que la neurogénesis hipocampal adulta ayuda a separar eficientemente unidades de información y participa activamente en la codificación temporal y en la memoria episódica (Kempermann, Krebs y Fabel, 2008). De hecho, la separación de patrones es una de las funciones que más se ha asociado a la neurogénesis, y es la habilidad de distinguir información separada en el tiempo, frecuentemente durante aprendizaje emocional (Kempermann, 2012; Snyder y Cameron, 2012). Gracias a este tipo de neurogénesis la red hipocampal puede adaptarse a niveles de novedad y complejidad a lo largo de la vida, e integrar información relevante y nueva en una representación aprendida (Garthe, Behr y Kempermann, 2009). Por lo tanto, parece que la neurogéne- 
sis adulta en el hipocampo favorece la adaptación a entornos cambiantes (Kempermann, 2012). Otro de los papeles que se han asociado a la neurogénesis es la regulación de respuestas de estrés (Snyder, Soumier, Brewer, Pickel y Cameron, 2011), amortiguando sus efectos nocivos (Surget et al., 2011); lo cual encaja con el hecho de que existen niveles altos de receptores de glucocorticoides en el hipocampo (y en células progenitoras) y su implicación en la retroalimentación negativa del eje hipotalámico-hipofisario-adrenal (Jacobson y Sapolsky, 1991), siendo requeridas las nuevas neuronas en la expresión normal del componente endocrino de la respuesta al estrés (CastillaOrtega et al., 2011). A su vez, recientemente se ha planteado una posible implicación de la neurogénesis en la formación de memoria emocional (Lee, Reif y Schmitt, 2013), tales como la formación de miedo condicionado y su extinción (Kirby et al., 2012) y ha sido relacionado su disfunción con algunas patologías como la depresión (Lee, Reif y Schmitt, 2013).

\section{Papel de la neurogénesis hipocampal adulta en el envejeci- miento}

Demostrar una relación causal entre el nivel de neurogénesis hipocampal adulta y el curso del envejecimiento cognitivo requiere demostrar que reducciones en la neurogénesis deterioran el proceso mientras que manipulaciones que incrementen la neurogénesis facilitarían el envejecimiento saludable, así como que los cambios en neurogénesis y funciones cognitivas durante el envejecimiento discurran de forma paralela.

A pesar de que la plasticidad neural continúa y no cesa a lo largo del ciclo vital, y como forma de tal la neurogénesis, en líneas generales en la literatura científica se observa un notable descenso de la neurogénesis hipocampal adulta en individuos de edad avanzada, de modo que representa tan solo una fracción de la tasa observada en la juventud (revisado en CouillardDesprés, Iglseder y Aigner, 2011). Concretamente, los procesos neurogénicos afectados negativamente por la edad son la proliferación de nuevas neuronas y la migración de las mismas por enlentecimiento, mientras que no se han hallado cambios significativos en cuanto a la supervivencia (Dapreau y Abrous, 2008; Encinas y Sierra, 2012). Esta disminución en los niveles de neurogénesis a edades avanzadas puede ser debido a un descenso en el número de precursores, reducción de la actividad proliferativa de los precursores, descenso en la proporción de células generadas las primeras semanas de maduración, o diferenciación sesgada hacia un fenotipo glial (Dapreau y Abrous, 2008). Sin embargo, dicho descenso no es invariable e irreversible, puesto que al estar la neurogénesis íntimamente relacionada con cambios ambientales, además de las diferencias interindividuales debidas a experiencias tempranas, podría ser reactivada mediante las señales adecuadas en cerebros viejos.

Múltiples estudios han lesionado las zonas neurogénicas utilizando radiación, agentes citoestáticos/citotóxicos o transgénicamente y han hallado deficiencias en el aprendizaje y la memoria (revisado en Lazarov y Marr, 2013). No obstante, la evidencia hasta la fecha no deja de ser controvertida y, aunque existen numerosos datos que implican el papel de la neurogénesis en diferentes tareas cognitivas (véase apartado 3), esta relación parece no ser unívoca e incluso puede diferir entre especies (Lazarov y Marr, 2013). Además, existen diferencias interindividuales de forma que ratas envejecidas con una tasa de proliferación neuronal del 15\% respecto al grupo de ratas jóvenes pueden mantener un índice de aprendizaje comparable al observado en animales jóvenes (Bizon, Lee y Gallagher, 2004). Sin embargo, se ha encontrado tanto en ratas (Drapeau et al., 2003), en monos (Aizawa, Ageyama, Yokoyama y Hisatsune, 2009) como en humanos (Coras et al., 2010) que la ejecución en tareas de memoria espacial correlaciona positivamente y predice los niveles de neurogénesis hipocampal en parámetros como el número de células proliferantes y de nuevas neuronas.

Por lo tanto, envejecimiento y neurogénesis parecen ser procesos paralelos, ya que en ambos quedan afectados determinados factores de forma que durante el envejecimiento los reguladores positivos de la neurogénesis tienden a disminuir, mientras que los inhibidores de la neurogénesis aumentan (Drapeau y Abrous, 2008). Reguladores positivos de la neurogénesis son: el ejercicio, la exposición al ambiente enriquecido, el aprendizaje, los antidepresivos, las hormonas sexuales, los shocks electroconvulsivos y la dieta, mientras que el estrés, la privación de sueño, la inflamación y la exposición crónica al abuso de drogas regulan la neurogénesis negativamente (Eisch et al., 2008; Lee et al., 2013; Lucassen et al., 2010; Stangl y Thuret, 2009).

Durante el envejecimiento, en hombres es común la deficiencia de andrógenos debido a la edad (Shelton y Rajfer, 2012) y en mujeres la concentración de estrógenos empieza a disminuir en torno a dos años antes del último ciclo menstrual. Los cambios hormonales asociados a la menopausia se han asociado al deterioro cognitivo relativo a la edad (Henderson, 2011). En este sentido, es un hecho ampliamente constatado que las hormonas gonadales afectan la plasticidad hipocampal (Korosi et al., 2012) y parecen regular también la generación de nuevas neuronas. Respecto a los andrógenos, se ha demostrado que la testosterona y su metabolito dihidrotestosterona incrementan la neurogénesis hipocampal favoreciendo la supervivencia de nuevas neuronas por medio de un mecanismo andrógenodependiente (Wainwright y Galea, 2013). En el caso de los estrógenos, estudios tanto in vitro como in vivo han encontrado que inducen un aumento de neurogénesis (Lee et al., 2013); en concreto de células tipo II proliferantes que dan lugar a un mayor número de neuronas inmaduras en el hipocampo de hembras (Galea, 2008) y además favorecen la supervivencia de las nuevas neuronas (Wainwright y Galea, 2013).

Como se ha indicado anteriormente, el estrés es uno de los factores que afecta negativamente la neurogénesis hipocampal adulta. Mientras que las hormonas asociadas al estrés inhiben tanto la proliferación de células como la supervivencia y diferenciación de las nuevas neuronas, causan atrofia hipocampal y 
por consiguiente perjudican el aprendizaje y la memoria, asociándose la exposición prolongada a altos niveles de corticosterona a lo largo de la vida del animal con el daño permanente en la proliferación de nuevas neuronas en animales envejecidos (Drapreau y Abrous, 2008). Sin embargo, el ejercicio moderado puede contrarrestar este efecto al mejorar la ejecución cognitiva e incrementar la neurogénesis (Wong y Herbert, 2004; Yau, Lau y So, 2011). Así pues, este deterioro de la neurogénesis hipocampal que tiene lugar durante el envejecimiento no es irreversible, pudiendo contrarrestarse con la exposición a factores que modulan positivamente la neurogénesis, como el ejercicio y el ambiente enriquecido.

Recientemente, Curlik y Shors (2013) encontraron que el entrenamiento tanto físico como mental aumenta significativamente la creación de nuevas neuronas y se ha asociado con salud mental. En un estudio más antiguo, van Praag et al. (2005) encontraron que el ejercicio voluntario mejora de la adquisición y la retención de memoria espacial en ratas envejecidas. En el mismo sentido, Speisman et al. (2013) informaron que el ejercicio diario mejora la memoria, estimula la neurogénesis hipocampal y modula la respuesta inmunológica en ratas envejecidas. Al igual que con el ejercicio, el ambiente enriquecido también ha atraído la atención de investigadores para suplir el deterioro cognitivo asociado a la edad (Koehl et al., 2011). Tanto la exposición a corto plazo como a largo plazo a un ambiente enriquecido impiden el decaimiento en la neurogénesis y mejoran la ejecución en la piscina de Morris (Kempermann y Gage, 2002; Kempermann, Kuhn y Gage, 1998; Kempermann et al., 2002). Estos efectos pueden ser mediados por factores neurotróficos. Por un lado, están implicados directamente en el funcionamiento normal de la plasticidad hipocampal y regulan positivamente la neurogénesis en esta estructura (Fournier y Duman, 2012; Lee y Son, 2009). Por otro lado, se han observado niveles reducidos de estos factores (BDNF y VEGF) a edades avanzadas (Jessberg y Gage, 2008). Zeng et al. (2011) encontraron que incrementando la expresión de BDNF en ratas se puede restaurar la plasticidad hipocampal y revertir el deterioro cognitivo asociado a la edad.

\section{Conclusión}

Aunque se ha descrito una gran variabilidad, el envejecimiento normal está frecuentemente asociado a un descenso tanto en neurogénesis como en rendimiento en tareas de aprendizaje y memoria dependientes de hipocampo (Lee, Clemenson y Gage, 2012). Por otro lado, las intervenciones que facilitan o deterioran la ejecución en edades avanzadas ejercen un efecto paralelo sobre la generación de nuevas neuronas hipocampales. Así, aunque no es fácil establecer una relación causal entre la neurogénesis hipocampal adulta y el desarrollo cognitivo, ya que los estudios de lesión ponen entredicho que sea un prerrequisito esencial (Lazarov y Marr, 2013), el conjunto de la información revisada permite concluir que existe una relación facilitadora entre neurogénesis hipocampal adulta y la eje- cución en tareas de aprendizaje y memoria dependientes del hipocampo durante el envejecimiento. Dicha relación podría ser tanto directa como indirecta ejerciendo efectos moduladores por medio de terceros factores, tales como la plasticidad sináptica hipocampal (Couillard-Després et al., 2012). Por ello, nuevos avances en el conocimiento de esta relación podrán suponer avances en salud mental y calidad de vida de esta ingente población. De este modo, conociendo los factores que favorecen la neurogénesis que pueden suplir ese descenso natural de las nuevas neuronas, varios autores señalan la importancia de fomentar variables como el ejercicio mental y físico para contribuir por medio de esta base neurobiológica al mantenimiento de funciones cognitivas e incluso prevenir o retrasar la aparición de trastornos neurodegenerativos (Koehl y Abrous, 2011; Lee et al., 2012; Park y Bischof, 2013). Asimismo, otro tipo de abordaje sería el farmacológico, en el cual por ejemplo se ha observado en roedores que una estimulación de la neurogénesis por parte de potenciadores cognitivos como el ginseng (Qiao et al., 2005), la modulación de receptores LPA (CastillaOrtega et al., 2012) o la supresión de receptores tipo Toll (Okun et al., 2010) se asocia también al rendimiento en varias tareas de aprendizaje.

\section{Referencias}

1. Aizawa, K., Ageyama, N., Yokoyama, C. y Hisatsune, T. (2009). Age-dependent alteration in hippocampal neurogenesis correlates with learning performance of macaque monkeys. Experimental Animals, 58, 403-407. http:// dx.doi.org/10.1538/expanim.58.403

2. Alexander, G. E., Ryan, L., Bowers, D., Foster, T. C., Bizon, J. L., Geldmacher, D. S. y Glisky, E. L. (2012). Characterizing cognitive aging in humans with links to animal models. Frontiers in Aging Neuroscience, 4, 21. http://dx.doi.org/10.3389/fnagi.2012.00021

3. Altman, J. y Das, G. D. (1965). Autoradiographic and histological evidence of postnatal hippocampal neurogenesis in rats. Journal of Comparative Neurology, 124, 319-336. http://dx.doi.org/10.1002/cne.901240303

4. Ball, M. J. (1977). Neuronal loss, neurofibrillary tangles and granulovacuolar degeneration in the hippocampus with ageing and dementia. A quantitative study. Acta Neuropathologica, 37, 111-118. http://dx.doi.org/10.1007/ BF00692056

5. Barnes, C. A. (1994). Normal aging: regionally specific changes in hippocampal synaptic transmission. Trends in Neuroscience, 17, 13-18. http://dx.doi.org/10.1016/01662236(94)90029-9

6. Bizon, J. L., Lee, H. J. y Gallagher, M. (2004). Neurogenesis in a rat model of age-related cognitive decline. Aging Cell, 3, 227-234. http://dx.doi.org/10.1111/j.14749728.2004.00099.x

7. Brandt, M. D., Jessberger, S., Steiner, B., Kronenberg, G., Reuter, K., Bick-Sander, A., von der Behrens, W. y 
Kempermann, G. (2003). Transient calretinin expression defines early postmitotic step of neuronal differentiation in adult hippocampal neurogenesis of mice. Molecular and Cellular Neurosciences, 24, 603-613. http://dx.doi. org/10.1016/S1044-7431(03)00207-0

8. Brickman, A. M., Meier, I. B., Korgaonkar, M. S., Provenzano, F. A., Grieve, S. M., Siedlecki, K. L., Wasserman, B. T., Williams, L. M. y Zimmerman, M. E. (2012). Testing the white matter retrogenesis hypothesis of cognitive aging. Neurobiology of Aging, 33, 1699-1715. http:// dx.doi.org/10.1016/j.neurobiolaging.2011.06.001

9. Brody, H. (1955). Organization of the cerebral cortex. III. A study of aging in the human cerebral cortex. Journal of Comparative Neurology, 102, 511-516. http://dx.doi. org/10.1002/cne.901020206

10. Burke, S. N. y Barnes, C. A. (2006). Neural plasticity in the ageing brain. Nature Reviews Neuroscience, 7, 30-40. http://dx.doi.org/10.1038/nrn1809

11. Burke, S. N., Wallace, S. N., Uprety, A. R. y Barnes, C. (2010) Pattern separation deficits may contribute to ageassociated recognition memory impairments. Behavioral Neuroscience, 124, 559-573. http://dx.doi.org/10.1037/ a0020893

12. Cansino, S. (2009). Episodic memory decay along the adult lifespan: a review of behavioral and neurophysiological evidence. International Journal of Psychophysiology, 71, 64-69. http://dx.doi.org/10.1016/j.ijpsycho.2008.07.005

13. Caserta, M. T., Bannon, Y., Fernandez, F., Giunta, B., Schoenberg, M. R. y Tan, J. (2009). Normal brain aging clinical, immunological, neuropsychological, and neuroimaging features. International Review of Neurobiology, 84, 1-19. http://dx.doi.org/10.1016/S0074-7742(09)00401-2

14. Castilla-Ortega, E., Hoyo-Becerra, C., Pedraza, C., Chun, J., Rodríguez De Fonseca, F., Estivill-Torrús, G. y Santín, L. J. (2011). Aggravation of chronic stress effects on hippocampal neurogenesis and spatial memory in LPA $\square$ receptor knockout mice. PLoS One, 6, e25522. http:// dx.doi.org/10.1371/journal.pone.0025522

15. Castilla-Ortega, E., Pedraza, C., Chun, J., de Fonseca, F. R., Estivill-Torrús, G. y Santín, L. J. (2012). Hippocampal c-Fos activation in normal and LPA $\square$-null mice after two object recognition tasks with different memory demands. Behavioural Brain Research, 232, 400-405. http://dx.doi. org/10.1016/j.bbr.2012.04.018

16. Chawla, M. K. y Barnes, C. A. (2007). Hippocampal granule cells in normal aging: insights from electrophysiological and functional imaging experiments. Progress in Brain Research, 163, 661-678. http://dx.doi.org/10.1016/S00796123(07)63036-2

17. Coras, R., Siebzehnrubl, F. A., Pauli, E., Huttner, H. B., Njunting, M., Kobow, K., Villmann, C., Hahnen, E., Neuhuber, W., Weigel, D., Buchfelder, M., Stefan, H., Beck, H., Steindler, D. A. y Blumcke, I. (2010). Low prolifera- tion and differentiation capacities of adult hippocampal stem cells correlate with memory dysfunction in humans. Brain, 133, 3359-3372. http://dx.doi.org/10.1093/brain/ awq215

18. Couillard-Després, S., Iglseder, B. y Aigner, L. (2011). Neurogenesis, cellular plasticity and cognition: the impact of stem cells in the adult and aging brain - A mini-review. Gerontology, 57, 559-564. http://dx.doi. org/10.1159/000323481

19. Craik, F. I. y Rose, N. S. (2012). Memory encoding and aging: a neurocognitive perspective. Neuroscience and Biobehavioral Reviews, 36, 1729-1739. http://dx.doi. org/10.1016/j.neubiorev.2011.11.007

20. Curlik, D. M. 2nd y Shors, T. J. (2013). Training your brain: Do mental and physical (MAP) training enhance cognition through the process of neurogenesis in the hippocampus? Neuropharmacology, 64, 506-514. http:// dx.doi.org/10.1016/j.neuropharm.2012.07.027

21. Drapeau, E. y Abrous D. N. (2008). Stem cell review series: role of neurogenesis in age-related memory disorders. Aging Cell, 7, 569-589. http://dx.doi.org/10.1111/ j.1474-9726.2008.00369.x

22. Drapeau, E., Mayo, W., Aurousseau, C., Le Moal, M., Piazza, P. V. y Abrous, D. N. (2003). Spatial memory performances of aged rats in the water maze predict levels of hippocampal neurogenesis. Proceedings of the National Academy of Sciences, 100, 14385-14390. http://dx.doi. org/10.1073/pnas.2334169100

23. Ehninger, D. y Kempermann, G. (2007). Neurogenesis in the adult hippocampus. Cell and Tissue Research, 331, 243-250. http://dx.doi.org/10.1007/s00441-007-0478-3

24. Eisch, A. J., Cameron, H. A., Encinas, J. M., Meltzer, L. A. y Ming, G. L. (2008). Adult neurogenesis, mental health, and mental illness: hope or hype? Journal of Neuroscience, 28, 11785-11791. http://dx.doi.org/10.1523/ JNEUROSCI.3798-08

25. Encinas, J. M. y Sierra, A. (2012). Neural stem cell deforestation as the main force driving the age-related decline in adult hippocampal neurogenesis. Behavioural Brain Research, 227, 433-439. http://dx.doi.org/10.1016/j. bbr.2011.10.010

26. Erickson, K. I., Miller, D. L. y Roecklein, K. A. (2012). The aging hippocampus: interactions between exercise, depression, and BDNF. The Neuroscientist, 18, 82-97. http://dx.doi.org/10.1177/1073858410397054

27. Eriksson, P. S., Perfilieva, E., Bjork-Eriksson, T., Alborn, A. M., Nordborg, C., Peterson, D. A y Gage, F. H. (1998). Neurogenesis in the adult human hippocampus. Nature Medicine, 4, 1313-1317. http://dx.doi.org/10.1038/3305

28. Foster, T. C. (2012). Challenges and opportunities in characterizing cognitive aging across species. Frontiers in Aging Neuroscience, 4, 1-2. http://dx.doi.org/10.3389/ fnagi.2012.00006 
29. Gage, F. H. (2000). Mammalian neural stem cells. Science, 287, 1433-1438. http://dx.doi.org/10.1126/science. 287.5457 .1433

30. Gallagher, M. y Rapp, P. R. (1997). The use of animal models to study the effects of aging on cognition. Annual Review of Psychology, 48, 339-370. http://dx.doi. org/10.1146/annurev.psych.48.1.339

31. Gallo, M., Valouskova, V. y Cándido, A. (1997). Fetal hippocampal transplants restore conditioned blocking in rats with dorsal hippocampal lesions: effect of age. Behavioral Brain Research, 88, 67-74. http://dx.doi.org/10.1016/ S0166-4328(97)02311-5

32. Gámiz, F. y Gallo, M. (2011). Taste learning and memory: a window on the study of brain aging. Frontiers in Systems Neuroscience, 5, 91-97. http://dx.doi.org/10.3389/ fnsys.2011.00091

33. Gámiz, F. y Gallo, M. (2012). Spontaneous object recognition memory in aged rats: complexity versus similarity. Learning and Memory, 19, 444-448. http://dx.doi. org/10.1101/lm.027003.112

34. Garthe, A., Behr, J. y Kempermann, G. (2009). Adult generated hippocampal neurons allow the flexible use of spatially precise learning strategies. PLoS ONE, 4, e5464. http://dx.doi.org/10.1371/journal.pone.0005464

35. Geinisman, Y., de Toledo-Morrell, L., Morrell, F., Persina, I. S. y Rossi, M. (1992). Age-related loss of axospinous synapses formed by two afferent systems in the rat dentate gyrus as revealed by the unbiased stereological dissector technique. Hippocampus, 2, 437-444. http://dx.doi. org/10.1002/hipo.450020411

36. Geinisman, Y., Ganeshina, O., Yoshida, R., Berry, R. W., Disterhoft, J. F. y Gallagher, M. (2004). Aging, spatial learning, and total synapse number in the rat CA1 stratum radiatum. Neurobiology of Aging, 25, 407-416. http:// dx.doi.org/10.1016/j.neurobiolaging.2003.12.001

37. Goh, J. O. y Park, D. C. (2009). Neuroplasticity and cognitive aging: The scaffolding theory of aging and cognition. Restorative Neurology and Neuroscience, 27, 391-403. http://dx.doi.org/10.3233/RNN-2009-0493

38. Goldman, S. A. y Nottebohm, F. (1983). Neuronal production, migration, and differentiation in a vocal control nucleus of the adult female canary brain. Proceedings of the National Academy of Sciences of the United States of America, 80, 2390-2394. http://dx.doi.org/10.1073/ pnas.80.8.2390

39. Gross, C. G. (2000). Neurogenesis in the adult brain: Death of a dogma. Nature Reviews Neuroscience, 1, 67-73. http:// dx.doi.org/10.1038/35036235

40. Head, D., Rodrigue, K. M., Kennedy, K. M. y Raz, N. (2008). Neuroanatomical and cognitive mediators of age-related differences in episodic memory. Neuropsychology, 22, 491-507. http://dx.doi.org/10.1037/0894$\underline{4105.22 .4 .491}$
41. Hedden, T. y Gabrieli, J. D. (2004). Insights into the ageing mind: a view from cognitive neuroscience. Nature Reviews Neuroscience, 5, 87-96. http://dx.doi.org/10.1038/nrn1323

42. Henderson, V. W. (2011). Gonadal hormones and cognitive aging: a midlife perspective. Women's Health, 7, 81-93. http://dx.doi.org/10.2217/whe.10.87

43. Jacobson, L. y Sapolsky, R. (1991). The role of the hippocampus in feedback regulation of the hypothalamic-pituitary-adrenocortical axis. Endocrine Reviews, 12, 118-134. http://dx.doi.org/10.1210/edrv-12-2-118

44. Jagust, W. (2013). Vulnerable neural systems and the borderland of brain aging and neurodegeneration. Neuron, 77, 219-234. http://dx.doi.org/10.1016/j.neuron.2013.01.002

45. Jessberger, S. y Gage, F. H. (2008). Stem Cell-associated structural and functional plasticity in the aging hippocampus. Psychology and Aging, 23, 684-691. http://dx.doi. org/10.1037/a0014188

46. Junqué, C. y Jurado, M. A. (1994). Envejecimiento normal. En: Junqué, C. y Jurado, M. A. Envejecimiento y demencias (pp. 13-203). Barcelona: Martínez Roca.

47. Kemp, J., Després, O., Sellal, F. y Dufour, A. (2012). Theory of Mind in normal ageing and neurodegenerative pathologies. Ageing Research Reviews, 11, 199-221. http:// dx.doi.org/10.1016/j.arr.2011.12.001

48. Kempermann, G. (2012). New neurons for 'survival of the fittest'. Nature Reviews Neuroscience, 13, 727-736. http:// dx.doi.org/10.1038/nrn3319

49. Kempermann, G. y Gage, F. H. (2002). Genetic influence on phenotypic differentiation in adult hippocampal neurogenesis. Developmental Brain Research, 134, 1-12. http:// dx.doi.org/10.1016/S0165-3806(01)00224-3

50. Kempermann, G., Gast, D. y Gage, F. H. (2002). Neuroplasticity in old age: sustained fivefold induction of hippocampal neurogenesis by long-term environmental enrichment. Developmental Annals of Neurology, 52, 135143. http://dx.doi.org/10.1002/ana.10262

51. Kempermann, G., Jessberger, S., Steiner, B. y Kronenberg, G. (2004). Milestones of neuronal development in the adult hippocampus. Trends in Neurosciences, 27, 447-452. http://dx.doi.org/10.1016/j.tins.2004.05.013

52. Kempermann, G., Krebs, J. y Fabel, K. (2008). The contribution of failing adult hippocampal neurogenesis to psychiatric disorders. Current Opinion in Psychiatry, 21, 290-295. http://dx.doi.org/10.1097/YCO.0b013e3282fad375

53. Kempermann, G., Kuhn, H. G. y Gage, F. H. (1998). Experience-induced neurogenesis in the senescent dentate gyrus. The Journal of Neuroscience, 18, 3206-3212.

54. Kirby, E. D., Kirby, E. D., Friedman, A. R., Covarrubias, D., Ying, C., Sun, W. G., Goosens, K. A., Sapolsky, R. M. y Kaufer, D. (2012). Basolateral amygdala regulation of adult hippocampal neurogenesis and fear-related activation of newborn neurons. Molecular Psychiatry, 17, 527536. http://dx.doi.org/10.1038/mp.2011.71 
55. Koehl, M. y Abrous, D. N. (2011). A new chapter in the field of memory: adult hippocampal neurogenesis. The European Journal of Neuroscience, 33, 1101-1114. http:// dx.doi.org/10.1111/j.1460-9568.2011.07609.x

56. Koh, M. T., Haberman, R. P., Foti, S., McCown, T. J. y Gallagher, M. (2010). Treatment strategies targeting excess hippocampal activity benefit aged rats with cognitive impairment. Neuropsychopharmacology, 35, 10161025. http://dx.doi.org/10.1038/npp.2009.207

57. Koh, M. T., Rosenzweig-Lipson, S. y Gallagher, M. (2013). Selective GABA(A) $\alpha 5$ positive allosteric modulators improve cognitive function in aged rats with memory impairment. Neuropharmacology, 64, 145-152. http:// dx.doi.org/10.1016/j.neuropharm.2012.06.023

58. Korosi, A., Naninck, E. F., Oomen, C. A., Schouten, M., Krugers, H., Fitzsimons, C. y Lucassen, P. J. (2012). Earlylife stress mediated modulation of adult neurogenesis and behavior. Behavioural Brain Research, 227, 400-409. http://dx.doi.org/10.1016/j.bbr.2011.07.037

59. Lazarov, O. y Marr, R. A. (2013). Of mice and men: neurogenesis, cognition and Alzheimer's disease. Frontiers in Aging Neuroscience, 5, 43. http://dx.doi.org/10.3389/ fnagi.2013.00043

60. Lazarov, O., Mattson, M. P., Peterson, D. A., Pimplikar, S. W. y van Praag, H. (2010). When neurogenesis encounters aging and disease. Trends in Neurosciences, 33, 569-579. http://dx.doi.org/10.1016/j.tins.2010.09.003

61. Lee, E. y Son, H. (2009). Adult hippocampal neurogenesis and related neurotrophic factors. BMB Reports, 42, 239244. http://dx.doi.org/10.5483/BMBRep.2011.44.6.421

62. Lee, M. M., Reif, A. y Schmitt, A. G. (2013). Major depression: a role for hippocampal neurogenesis? Current Topics in Behavioral Neurosciences, 14, 153-179. http:// dx.doi.org/10.1007/7854 2012 226

63. Lee, S. W., Clemenson, G. D. y Gage, F. H. (2012). New neurons in an aged brain. Behavioural Brain Research, 227, 497-507. http://dx.doi.org/10.1016/j.bbr.2011.10.009

64. Lemaire, V., Koehl, M., Le Moal, M., y Abrous, D.N. (2000). Prenatal stress produces learning deficits associated with an inhibition of neurogenesis in the hippocampus. Proceedings of the National Academy of Sciences USA, 97, 11032-11037. http://dx.doi.org/10.1073/pnas.97.20.11032

65. Lemaire, V., Lamarque, S., Le Moal, M., Piazza, P.V. y Abrous, D.N. (2006). Postnatal stimulation of the pups counteracts prenatal stress-induced deficits in hippocampal neurogenesis. Biological Psychiatry, 59, 786-792. http://dx.doi.org/10.1016/j.biopsych.2005.11.009

66. Lister, J. P. y Barnes, C. A. (2009). Neurobiological changes in the hippocampus during normative aging. Archives of Neurology, 66, 829-833. http://dx.doi.org/10.1001/archneurol.2009.125

67. Lithfous, S., Dufour, A. y Després, O. (2013). Spatial navigation in normal aging and the prodromal stage of
Alzheimer's disease: Insights from imaging and behavioral studies. Ageing Research Reviews, 12, 201-213. http:// dx.doi.org/10.1016/j.arr.2012.04.007

68. Lucassen, P. J., Meerlo, P., Naylor, A. S., van Dam, A. M., Dayer, A. G., Fuchs, E., Oomen, C. A. y Czeh, B. (2010). Regulation of adult neurogenesis by stress, sleep disruption, exercise and inflammation: Implications for depression and antidepressant action. European Neuropsychopharmacology, 20, 1-17. http://dx.doi.org/10.1016/j. euroneuro.2009.08.003

69. Manrique, T., Morón, I., Ballesteros, M. A., Guerrero, R. M. y Gallo, M. (2007). Hippocampus, Ageing, and Taste Memories. Chemical Senses, 32, 111-117. http://dx.doi. org/10.1093/chemse/bj1042

70. Manrique, T., Morón, I., Ballesteros, M. A., Guerrero, R. M., Fenton, A. A. y Gallo, M. (2009). Hippocampus, aging, and segregating memories. Hippocampus, 19, 57-65. http://dx.doi.org/10.1002/hipo.20481

71. Masiulis, I., Sanghee, Y. y Eisch, A. J. (2011). The interesting interplay between interneurons and adult hippocampal neurogenesis. Molecular Neurobiology, 44, 287-302. http://dx.doi.org/10.1007/s12035-011-8207-z

72. Meaney, M. J., Aitken, D. H., Bhatnagar, S., y Sapolsky, R. M. (1991). Postnatal handling attenuates certain neuroendocrine, anatomical and cognitive dysfunction associated with aging in female rats. Neurobiology of Aging, 12, 31-38. http://dx.doi.org/10.1016/0197-4580(91)90036-J

73. Mendelsohn, A. R. y Larrick, J. W. (2012). Epigeneticmediated decline in synaptic plasticity during aging. Rejuvenation Research, 15, 98-101. http://dx.doi.org/10.1089/ rej.2012.1312

74. Milner, B. (2005). The medial temporal-lobe amnesic syndrome. The Psychiatric Clinics of North America, 28, 599611. http://dx.doi.org/10.1016/j.psc.2005.06.002

75. Morón, I y Gallo, M. (2007) Effect of previous taste experiences on taste neophobia in young-adult and aged rats. Physiology and Behavior, 90, 308-317. http://dx.doi. org/10.1016/j.physbeh.2006.09.036

76. Morón, I., Ballesteros, M. A., Cándido, A. y Gallo, M. (2002). Taste aversion learning and aging: A comparison with the effect of dorsal hippocampal lesions in rats. Physiological Research, 51, S21-S27.

77. Morón, I., Ballesteros, M. A., Valouskova, V. y Gallo, M. (2001). Conditioned blocking is re-established by neurotrasplantation in mature rats. NeuroReport, 12, 2297-2301.

78. Okun, E., Griffioen, K., Barak, B., Roberts, N. J., Castro, K., Pita, M.A., Cheng, A., Mughal, M. R., Wan, R., Ashery, U. y Mattson, M. P. (2010). Toll-like receptor 3 inhibits memory retention and constrains adult hippocampal neurogenesis. Proceedings of the National Academy of Sciences, 107, 15625-15630. http://dx.doi.org/10.1073/ pnas. 1005807107

79. Pannese, E. (2011). Morphological changes in nerve cells 
during normal aging. Brain Structure \& Function, 216, 85-89. http://dx.doi.org/10.1007/s00429-011-0308-y

80. Park, D. C. y Bischof, G. N. (2013). The aging mind: neuroplasticity in response to cognitive training. Dialogues in Clinical Neuroscience, 15, 109-119.

81. Park, D. C. y Reuter-Lorenz, P. (2009). The adaptive brain: aging and neurocognitive scaffolding. Annual Review of Psychology, 60, 173-196. http://dx.doi.org/10.1146/ annurev.psych.59.103006.093656

82. Patrylo, P. R. y Williamson, A. (2007). The effects of aging on dentate circuitry and function. Progress in Brain Research, 163, 679-696. http://dx.doi.org/10.1016/S00796123(07)63037-4

83. Qiao, C., Den, R., Kudo, K., Yamada, K., Takemoto, K., Wati, H. y Kanba, S. (2005). Ginseng enhances contextual fear conditioning and neurogenesis in rats. Neuroscience Research, 51, 31-38. http://dx.doi.org/10.1016/j. neures.2004.09.004

84. Reuter-Lorenz, P.A. y Lustig, C. (2005) Brain aging: reorganizing discoveries about the aging mind. Current Opinion in Neurobiology, 15, 245-251. http://dx.doi. org/10.1016/j.conb.2005.03.016

85. Rieckmann, A. y Bäckman, L. (2009). Implicit learning in aging: extant patterns and new directions. Neuropsychological Review, 19, 490-503. http://dx.doi.org/10.1007/ s11065-009-9117-y

86. Rosenzweig, E. S. y Barnes, C. A. (2003). Impact of aging on hippocampal function: plasticity, network dynamics, and cognition. Progress in Neurobiology, 69, 143-179. http://dx.doi.org/10.1016/S0301-0082(02)00126-0

87. Salat, D. H. (2011). The declining infrastructure of the aging brain. Brain Connectivity, 1, 279-293. http://dx.doi. org/10.1089/brain.2011.0056

88. Samson, R. D. y Barnes, C. A. (2013). Impact of aging brain circuits on cognition. European Journal of Neuroscience, 37, 1903-1915. http://dx.doi.org/10.1111/ejn.12183

89. Shelton, J. B. y Rajfer, J. (2012). Androgen deficiency in aging and metabolically challenged men. The Urologic Clinics of North America, 39, 63-75. http://dx.doi. org/10.1016/j.ucl.2011.09.007

90. Snyder, J. S. y Cameron, H. A. (2012). Could adult hippocampal neurogenesis be relevant for human behavior? Behavioural Brain Research, 227, 384-390. http://dx.doi. org/10.1016/j.bbr.2011.06.024

91. Snyder, J. S., Soumier, A., Brewer, M., Pickel, J. y Cameron, H. A. (2011). .Adult hippocampal neurogenesis buffers stress responses and depressive behaviour. Nature. 476, 458-461. http://dx.doi.org/10.1038/nature10287

92. Spreng, R. N., Wojtowicz, M. y Grady, Ch. L. (2010). Reliable differences in brain activity between young and old adults: a quantitative meta-analysis across multiple cognitive domains. Neuroscience and Biobehavioral Reviews, 34, 1178-1194. http://dx.doi.org/10.1016/j.neu- biorev.2010.01.009

93. Song, J., Christian, K. M., Ming, G. L. y Song, H. (2012). Modification of hippocampal circuitry by adult neurogenesis. Developmental Neurobiology, 72, 1032-1043. http:// dx.doi.org/10.1002/dneu.22014

94. Speisman, R. B., Kumar, A., Rani, A., Foster, T. C. y Ormerod, B. K. (2013). Daily exercise improves memory, stimulates hippocampal neurogenesis and modulates immune and neuroimmune cytokines in aging rats. Brain, Behavior, and Immunity, 28, 25-43. http://dx.doi. org/10.1016/j.bbi.2012.09.013

95. Stangl, D. y Thuret, S. (2009). Impact of diet on adult hippocampal neurogenesis. Genes \& Nutrition, 4, 271-282. http://dx.doi.org/10.1007/s12263-009-0134-5

96. Steffener, J. y Stern, Y. (2012). Exploring the neural basis of cognitive reserve in aging. Biochimica et Biophysica Acta, 1822, 467-473. http://dx.doi.org/10.1016/j. bbadis.2011.09.012

97. Surget, A., Tanti, A., Leonardo, E. D., Laugeray, A., Rainer, Q., Touma, C., Palme, R., Griebel, G., IbarguenVargas, Y., Hen, R. y Belzung, C. (2011). Antidepressants recruit new neurons to improve stress response regulation. Molecular Psychiatry, 16, 1177-1188. http://dx.doi. org/10.1038/mp.2011.48

98. Turner, G. R. y Spreng, R. N. (2012). Executive functions and neurocognitive aging: dissociable patterns of brain activity. Neurobiology of Aging, 33, 826, e1-13. http:// dx.doi.org/10.1016/j.neurobiolaging.2011.06.005

99. Van Praag, H., Shubert, T., Zhao, C. y Gage, F.H. (2005). Exercise enhances learning and hippocampal neurogenesis in aged mice. The Journal of Neuroscience, 25, 8680-8685. http://dx.doi.org/10.1523/JNEUROSCI.1731-05.2005

100. Varela-Nallar, L., Aranguiz, F. C., Abbott, A. C., Slater, P. G e Inestrosa, N. C. (2010). Adult hippocampal neurogenesis in aging and Alzheimer's disease. Birth Defects Research. Part C, Embryo Today: Reviews, 90, 284-296. http://dx.doi.org/10.1002/bdrc.20193

101. Wainwright, S. R. y Galea, L. A. (2013). The Neural Plasticity Theory of Depression: Assessing the roles of adult neurogenesis and PSA-NCAM within the hippocampus. Neural Plasticity, http://dx.doi.org/10.1155/2013/805497

102. Wang, M., Gamo, N. J., Yang, Y., Jin, L. E., Wang, X. J., Laubach, M., Mazer, J. A., Lee, D. y Arnsten, A. F. (2011). Neuronal basis of age-related working memory decline. Nature, 476, 210-223. http://dx.doi.org/10.1038/ nature 10243

103. Wilson, I. A., Ikonen, S., Gallagher, M., Eichenbaum, H. y Tanila, H. (2005). Age-associated alterations of hippocampal place cells are subregion specific. Journal of Neuroscience, 25, 6877-6886. http://dx.doi.org/10.1523/ JNEUROSCI.1744-05.2005

104. Wilson, I. A., Ikonen, S., McMahan, R. W., Gallagher, M., Eichenbaum, H. y Tanila, H. (2003). Place cell rigidity cor- 
relates with impaired spatial learning in aged rats. Neurobiology of Aging, 24, 297-305. http://dx.doi.org/10.1016/ S0197-4580(02)00080-5

105. Wong, E. Y. y Herbert, J. (2004). The corticoid environment: a determining factor for neural progenitors' survival in the adult hippocampus. The European Journal of Neuroscience, 20, 2491-2498. http://dx.doi.org/10.1111/j.14609568.2004.03717.x

106. Yau, S. Y., Lau, B. W. y So, K. F. (2011). Adult hippocampal neurogenesis: a possible way how physical exercise counteracts stress. Cell Transplantation, 20, 99-111. http:// dx.doi.org/10.3727/096368910X532846

107.Zeng, Y., Tan, M., Kohyama, J., Sneddon, M., Watson, J. B., Sun, Y. E. y Xie, C. W. (2011). Epigenetic enhancement of BDNF signaling rescues synaptic plasticity in aging. The Journal of Neuroscience, 31, 17800-17810. http:// dx.doi.org/10.1523/JNEUROSCI.3878-11.2011

Fecha de recepción: 15 de septiembre de 2013 Fecha de recepción de la versión modificada: 20 de octubre de 2013 Fecha de aceptación: 25 de octubre de 2013 